International Journal of Pattern Recognition and Artificial Intelligence

Vol. 22, No. 8 (2008) 1673-1682

(c) World Scientific Publishing Company

\title{
AUTHOR INDEX \\ Volume 22 (2008)
}

Abowd, G. D., see Yun, J.

22 (2008) 497-514

Ahmed, A. A. E., see Nazar, A.

22 (2008) 461-495

Al-Tahrawi, M. M. \& Zitar, R. A., Polynomial networks versus other techniques in text categorization

22 (2008) 295-322

Allahverdi, N., see Altun, A. A.

22 (2008) 585-600

Altun, A. A., Kocer, H. E. \& Allahverdi, N., Genetic algorithm based feature selection level fusion using fingerprint and iris biometrics

Amor, N. B., see Borchani, H.

Aslan, K., see Sahin, C.

Bai, X., Yang, X., Yu, D. \& Latecki, L. J., Skeleton-based shape classification using path similarity

Beltrán, N. H., see Bustos, M. A.

22 (2008) 585-600

22 (2008) 253-278

22 (2008) 973-985

22 (2008) 733-746

22 (2008) 1089-1119

Bertolami, R. \& Bunke, H., Integration of $n$-gram language models in multiple classifier systems for offline handwritten text line recognition

22 (2008) 1301-1321

Boccignone, G., Napoletano, P. \& Ferraro, M., Embedding diffusion in variational bayes: a technique for segmenting images

22 (2008) 811-827

22 (2008) 1015-1029

Boccignone, G., see Cagli, R. C.

22 (2008) 1241-1265 fuzzy learning for human skin detection

Borchani, H., Amor, N. B. \& Khalfallah, F., Learning and evaluating bayesian network equivalence classes from incomplete data

22 (2008) 253-278

22 (2008) 1461-1486

Boutalis, Y. S., see Papakostas, G. A.

22 (2008) 973-985

Brouwer, R. K., Clustering quality measures based on comparing the proximity matrices for the membership vectors and the objects

22 (2008) 1513-1538

Bunke, H., see Bertolami, R.

22 (2008) 1301-1321 
Burattini, E. \& Rossi, S., Periodic adaptive activation of behaviors in robotic systems

Bustos, M. A., Duarte-Mermoud, M. A. \& Beltrán, N. H., Nonlinear feature extraction using fisher criterion

22 (2008) 1089-1119

Cagli, R. C., Coraggio, P., Napoletano, P. \& Boccignone, G., What the draughtsman's hand tells the draughtsman's eye: a sensorimotor account of drawing

Cai, Z., see Jiang, L.

Calabrese, A., Mele, F., Sorgente, A., Talamo, O. \& Coppola, C., Three conceptual levels for the artifacts design

Cao, Q., see Ni, F.

Chan, A. P. F., Chan, P. P. K., Ng, W. W. Y., Tsang, E. C. C. \& Yeung, D. S., A novel feature grouping method for ensemble neural network using localized generalization error model

Chan, P. P. K., see Chan, A. P. F.

Chang, T.-W., see Huang, Y.-P.

Chang, T.-W., see Huang, Y.-P.

22 (2008) 1015-1029

22 (2008) 1121-1140

22 (2008) 1059-1072

22 (2008) 1647-1668

Chang, Y.-H., see Wang, J.-F.

Chang, Y.-J., Wang, R.-Z. \& Lin, J.-C., Hiding images using modified search-order coding and modulus function

Chavarriaga, R., see Millán, J. del R.

Chen, F., see Chen, Y.

Chen, Q., Wang, X., Su, P. \& Yao, Y., Auto adapted English pronunciation evaluation: a fuzzy integral approach

Chen, S., see Liu, W.

Chen, T.-Y., see Wang, J.-F.

Chen, Y., Chen, F., Yang, J. Y. \& Yang, M. Q., Ensemble voting system for multiclass protein fold recognition

Chen, Y.-R., see Huang, Y.-P.

Cheng, H. D., see Wang, L.

Cheng, S., see Shih, F. Y.

Chiang, T.-W., see Tsai, T.

Choi, T.-S., see Malik, A. S.

Chou, H.-L., see Tang, C.-Y.

22 (2008) 137-151

22 (2008) 137-151

22 (2008) 233-251

22 (2008) 711-732

22 (2008) 601-616

22 (2008) 1215-1240

22 (2008) 959-972

22 (2008) 747-763

22 (2008) 153-168

22 (2008) 1587-1598

22 (2008) 601-616

22 (2008) 747-763

22 (2008) 233-251

22 (2008) 1379-1401

22 (2008) 515-534

22 (2008) 765-781

22 (2008) 945-958

22 (2008) 783-805

22 (2008) 445-459

22 (2008) 515-534

Chuang, C.-F., see Shih, F. Y.

22 (2008) 1059-1072

Coppola, C., see Calabrese, A.

Coraggio, P., De Gregorio, M. \& Forastiere, M., Robot

navigation based on neurosymbolic reasoning over

landmarks

22 (2008) 1001-1014

Coraggio, P., see Cagli, R. C.

22 (2008) 1015-1029

Costa, A. H. R., see Bonventi Jr., W.

22 (2008) 1241-1265 
De Gregorio, M., see Coraggio, $\mathrm{P}$.

Ding, X., see Feng, X.

Ding, Y.-H., see Tang, C.-Y.

Dong, J., see Zhou, $\mathrm{H}$.

Du, S., see You, Q.

Duarte-Mermoud, M. A., see Bustos, M. A.

El Abed, H., see Mozaffari, S.

Erol, R., see Sahin, C.

Esposito, A., Stejskal, V. \& Smékal, Z., Cognitive role of speech pauses and algorithmic considerations for their processing

Faez, K., see Mozaffari, S.

Feng, X., Ding, X., Wu, Y. \& Wang, P. S. P., Classifier combination and its application in iris recognition

Feng, Z., see Lu, N.

Ferone, A., see Maddalena, L.

Ferraro, M., see Boccignone, G.

Ferreira, T. O., see Lima, P. M. V.

Ferrez, P. W., see Millán, J. del R.

Foggia, P., Percannella, G., Sansone, C. \& Vento, M., A graph-based algorithm for cluster detection

Forastiere, M., see Coraggio, $\mathrm{P}$.

França, F. M. G., see Lima, P. M. V.

Frucci, M., Perner, P. \& Sanniti di Baja, G.,

Case-based-reasoning for image segmentation

Fu, G., see Shih, F. Y.

Fu, Y.-Y., see Shih, F. Y.

$\mathrm{Fu}, \mathrm{Z}$., see Ni, F.

Galán, F., see Millán, J. del R.

Gao, L., see You, Q.

Gao, Q., see Gao, X.

Gao, X., Yue, H., Li, L. \& Gao, Q., Semantic-parsing based on semantic units theory - a new approach to natural languages processing

Gavrilova, M. L., see Luo, Y.

Ha, M.-H. \& Tian, X.-D., An improved algorithm of optical formula extraction with fuzzy classification

He, Q., Zhao, X.-R. \& Shi, Z.-Z., Minimal consistent subset for hyper surface classification method

Hiremath, P. S. \& Prabhakar, C. J., Symbolic factorial discriminant analysis for illumination invariant face recognition
22 (2008) 1001-1014

22 (2008) 617-638

22 (2008) 783-805

22 (2008) 279-294

22 (2008) 1283-1299

22 (2008) 1089-1119

22 (2008) 1323-1341

22 (2008) 973-985

22 (2008) 1073-1088

22 (2008) 1323-1341

22 (2008) 617-638

22 (2008) 1559-1586

22 (2008) 915-928

22 (2008) 811-827

22 (2008) 1031-1043

22 (2008) 959-972

22 (2008) 843-860

22 (2008) 1001-1014

22 (2008) 1031-1043

22 (2008) 829-842

22 (2008) 323-334

22 (2008) 335-345

22 (2008) 1647-1668

22 (2008) 959-972

22 (2008) 1283-1299

22 (2008) 1447-1460

22 (2008) 1447-1460

22 (2008) 555-584

22 (2008) 77-93

22 (2008) 95-108

22 (2008) 371-387 
Hu, J.-S. \& Su, T.-M., Flexible 3D object recognition framework using 2D views via a similarity-based aspect-graph approach

22 (2008) 1141-1169

Huang, B., see Jiang, L.

22 (2008) 1121-1140

Huang, C.-Y., see Liu, L.-M.

22 (2008) 347-365

Huang, Y.-P., Chang, T.-W. \& Sandnes, F. E., Efficient shape-based image retrieval based on gray relational analysis and association rules

22 (2008) 711-732

Huang, Y.-P., Chang, T.-W., Chen, Y.-R. \& Sandnes, F. E., A back propagation based real-time license plate recognition system

Huang, Y.-P., see Tsai, T.

Hung, D. C. D., see Liu, L.-M.

Imiya, A., see Ohnishi, N.

Ji, Z., see Liu, W.

Jiang, L., Wang, D., Zhang, H., Cai, Z. \& Huang, B., Using instance cloning to improve naive bayes for ranking

Jin, C.-X., see Li, F.-C.

Jin, F., Song, S.-J. \& Wu, C., A simulated annealing based beam search algorithm for the flow-shop scheduling problem Jin, Y., see Zhang, C.

Jogan, M., see Štimec, A.

Kamel, N. S. \& Sayeed, S., SVD-based signature verification technique using data glove

Kang, S., see Lee, S.-W.

Khalfallah, F., see Borchani, H.

Kitani, K. M., Sato, Y. \& Sugimoto, A., Recognizing overlapped human activities from a sequence of primitive actions via deleted interpolation

22 (2008) 1343-1362

Kitani, K. M., Sato, Y. \& Sugimoto, A., Recovering the basic structure of human activities from noisy video-based symbol strings

Kocer, H. E., see Altun, A. A.

22 (2008) 1621-1646

22 (2008) 585-600

22 (2008) 883-897

22 (2008) 1461-1486

22 (2008) 411-429

Krzyżak, A., see Rokita, J.

Kuo, W.-F., Lin, C.-Y. \& Sun, Y.-N., Region similarity relationship between watershed and penalized fuzzy hopfield neural network algorithms for brain image segmentation

Lai, L. L., see Lu, Y.

Latecki, L. J., see Bai, X.

22 (2008) 233-251

22 (2008) 765-781

22 (2008) 347-365

22 (2008) 929-944

22 (2008) 1587-1598

22 (2008) 1121-1140

22 (2008) 17-32

22 (2008) 65-75

22 (2008) 1427-1445

22 (2008) 639-665

22 (2008) 431-443

22 (2008) 1171-1186

22 (2008) 253-278

Lee, S.-W., Kang, S. \& Lee, S.-W., A walking guidance system for the visually impaired

22 (2008) 1403-1425

22 (2008) 55-64

22 (2008) 733-746

22 (2008) 1171-1186 
Lee, S.-W., see Lee, S.-W.

22 (2008) 389-410

Lee, S.-W., see Lee, S.-W.

22 (2008) 1171-1186

Lee, S.-W., Wang, P. S. P., Yanushkevich, S. N. \& Lee, S.-W., Noniterative 3D face reconstruction based on photometric stereo

22 (2008) 389-410

Leonardis, A., see Štimec, A.

22 (2008) 639-665

Lew, E., see Millán, J. del R.

22 (2008) 959-972

Li, F.-C., Jin, C.-X. \& Yue, P.-X., Operation for a new kind of fuzzy genetic algorithm based on the transformation of the principle index

Li, L., see Gao, X.

Li, X., see Zhang, T.

Li, X., see Zhou, H.

Lima, P. M. V., Morveli-Espinoza, M. M. M., Pereira, G. C., Ferreira, T. O. \& França, F. M. G., Logical reasoning via satisfiability mapped into energy functions

22 (2008) 1031-1043

Lin, C.-H., Lin, Y.-Y. \& Sandnes, F. E., Integrity protection of binary text document images based on image topologies

Lin, C.-L., see Wang, J.-F.

Lin, C.-Y., see Kuo, W.-F.

Lin, F., see Zhou, H.

Lin, J.-C., see Chang, Y.-J.

Lin, X., see Lu, Y.

Lin, Y.-Y., see Lin, C.-H.

Liu, H., see Liu, M.-S.

Liu, L.-M., Huang, C.-Y. \& Hung, D. C. D., A directional approach to fingerprint classification

22 (2008) 17-32

22 (2008) 1447-1460

22 (2008) 667-690

22 (2008) 279-294

22 (2008) 1599-1620

22 (2008) 601-616

22 (2008) 1403-1425

22 (2008) 279-294

22 (2008) 1215-1240

22 (2008) 55-64

22 (2008) 1599-1620

22 (2008) 33-54

22 (2008) 347-365

Liu, J.-M. \& Wang, J.-H., Parameters optimize method based on genetic and simulated annealing algorithms

Liu, M.-S., Liu, H. \& Zheng, J.-W., A strategy for securing APDU transmission

22 (2008) 169-181

22 (2008) 33-54

22 (2008) 279-294

Liu, T., see Zhou, H.

Liu, W., Yuan, K., Wu, J., Ye, D., Ji, Z. \& Chen, S., Combining generalized $\mathrm{NMF}$ and discriminative mixture models for classification of gene expression data

22 (2008) 1587-1598

Lu, N. \& Feng, Z., Accumulative intersection space based corner detection algorithm

Lu, S.-X., see Wang, X.-Z.

22 (2008) 1559-1586

22 (2008) 109-120

Lu, Y., Yu, M., Lai, L. L. \& Lin, X., A new fuzzy neural network strategy of insulators contamination detection for power system transmission line

22 (2008) 55-64 
Luo, Y., Gavrilova, M. L. \& Wang, P. S. P., Facial metamorphosis using geometrical methods for biometric applications

Ma, W.-M., Tang, S.-H. \& Wang, K., Competitive analysis for the most reliable path problem with online and fuzzy uncertainties

22 (2008) 195-206

Maddalena, L., Petrosino, A. \& Ferone, A., Object motion detection and tracking by an artificial intelligence approach

Malik, A. S. \& Choi, T.-S., Effect of noise and source illumination on 3D shape recovery

Märgner, V., see Mozaffari, S.

Mele, F., see Calabrese, A.

Mertzios, B. G., see Papakostas, G. A.

Millán, J. del R., Ferrez, P. W., Galán, F., Lew, E. \& Chavarriaga, R., Non-invasive brain-machine interaction

Morveli-Espinoza, M. M. M., see Lima, P. M. V.

Mozaffari, S., Faez, K., Märgner, V. \& El Abed, H., Two-stage lexicon reduction for offline arabic handwritten word recognition

22 (2008) 1323-1341

Mukundan, R., Fast computation of geometric moments and invariants using schlick's approximation

Nagurka, M. L., see Wang, J.-F.

Napoletano, P., see Boccignone, G.

Napoletano, P., see Cagli, R. C.

Nazar, A., Traoré, I. \& Ahmed, A. A. E., Inverse biometrics for mouse dynamics

Ng, W. W. Y., see Chan, A. P. F.

Ng, W. W. Y., Yeung, D. S. \& Tsang, E. C. C., The localized generalization error model for single layer perceptron neural network and sigmoid support vector machine

22 (2008) 121-135

Ni, F., Fu, Z., Cao, Q. \& Zhao, Y., A new method for facial features quantification of caricature based on self-reference model

22 (2008) 1647-1668

Oberhoff, D. \& Kolesnik, M., Neural object recognition by hierarchical appearance learning

Ogulata, S. N., see Sahin, C.

22 (2008) 883-897

22 (2008) 973-985

Ohnishi, N. \& Imiya, A., Independent components of optical flow in a multiresolution image sequence

22 (2008) 929-944

Panagiotakis, C., Ramasso, E., Tziritas, G., Rombaut, M. \& Pellerin, D., Shape-based individual/group detection for sport videos categorization

22 (2008) 1187-1213

Pang, Y., see Zhou, H.

22 (2008) 279-294 
Papakostas, G. A., Boutalis, Y. S., Koulouriotis, D. E. \& Mertzios, B. G., Fuzzy cognitive maps for pattern recognition applications

Papamarkos, N., see Saragiotis, P.

Pellerin, D., see Panagiotakis, C.

Percannella, G., see Foggia, P.

Pereira, G. C., see Lima, P. M. V.

Perner, P., see Frucci, M.

Petrosino, A., see Maddalena, L.

Prabhakar, C. J., see Hiremath, P. S.

Raducanu, B. \& Vitrià, J., Face recognition by artificial vision systems: a cognitive perspective

Ramasso, E., see Panagiotakis, C.

Rendek, J. \& Wendling, L., On determining suitable subsets of decision rules using choquet integral

Rodríguez-Sánchez, A. J., see Rothenstein, A. L.

Rokita, J., Krzyżak, A. \& Suen, C. Y., Multimodal biometrics by face and hand images taken by a cell phone camera

Rombaut, M., see Panagiotakis, C.

Rossi, S., see Burattini, E.

Rothenstein, A. L., Rodríguez-Sánchez, A. J., Simine, E. \& Tsotsos, J. K., Visual feature binding within the selective tuning attention framework

Ruan, Q., see Zhang, C.

Ryu, J., see Yun, J.

Sahin, C., Ogulata, S. N., Aslan, K., Bozdemir, H. \& Erol, R., A neural network-based classification model for partial epilepsy by EEG signals

Sandnes, F. E., see Huang, Y.-P.

Sandnes, F. E., see Huang, Y.-P.

Sandnes, F. E., see Lin, C.-H.

Sanniti di Baja, G., see Frucci, M.

Sanromà, G., see Serratosa, F.

Sansone, C., see Foggia, P.

Saragiotis, P. \& Papamarkos, N., Local skew correction in documents

Sarkar, B., An efficient method for near-optimal polygonal approximation based on differential evolution

Sato, Y., see Kitani, K. M.

Sato, Y., see Kitani, K. M.

Sayeed, S., see Kamel, N. S.

Serratosa, F. \& Sanromà, G., A fast approximation of the earth-movers distance between multidimensional histograms
22 (2008) 1461-1486

22 (2008) 691-710

22 (2008) 1187-1213

22 (2008) 843-860

22 (2008) 1031-1043

22 (2008) 829-842

22 (2008) 915-928

22 (2008) 371-387

22 (2008) 899-913

22 (2008) 1187-1213

22 (2008) 207-232

22 (2008) 861-881

22 (2008) 411-429

22 (2008) 1187-1213

22 (2008) 987-999

22 (2008) 861-881

22 (2008) 1427-1445

22 (2008) 497-514

22 (2008) 973-985

22 (2008) 233-251

22 (2008) 711-732

22 (2008) 1599-1620

22 (2008) 829-842

22 (2008) 1539-1558

22 (2008) 843-860

22 (2008) 691-710

22 (2008) 1267-1281

22 (2008) 1343-1362

22 (2008) 1621-1646

22 (2008) 431-443

22 (2008) 1539-1558 
Shi, Z.-Z., see He, Q.

22 (2008) 95-108

Shih, F. Y. \& Fu, G., Decision combination of multiple classifiers

22 (2008) 323-334

Shih, F. Y., Cheng, S., Chuang, C.-F. \& Wang, P. S. P., Extracting faces and facial features from color images

22 (2008) 515-534

Shih, F. Y., Chuang, C.-F. \& Wang, P. S. P., Performance comparisons of facial expression recognition in JAFFE database

22 (2008) 445-459

Shih, F. Y. \& Fu, Y.-Y., Approximate image quality measure in low-dimensional domain based on random projection

Simine, E., see Rothenstein, A. L.

Smékal, Z., see Esposito, A.

Song, S.-J., see Jin, F.

Sorgente, A., see Calabrese, A.

Srihari, S. N. \& Srinivasan, H., Comparison of ROC and likelihood decision methods in automatic fingerprint verification

22 (2008) 535-553

22 (2008) 535-553

22 (2008) 1073-1088

Stejskal, V., see Esposito, A.

Štimec, A., Jogan, M. \& Leonardis, A., Unsupervised learning of a hierarchy of topological maps using omnidirectional images

22 (2008) 335-345

22 (2008) 861-881

22 (2008) 1073-1088

22 (2008) 65-75

22 (2008) 1059-1072

Stock, O., Strapparava, C. \& Valitutti, A., Ironic expressions and moving words

Strapparava, C., see Stock, O.

$\mathrm{Su}, \mathrm{K} .-\mathrm{P}$., see Wang, J.-F.

$\mathrm{Su}, \mathrm{P}$., see Chen, Q.

$\mathrm{Su}, \mathrm{T} .-\mathrm{M} .$, see Hu, J.-S.

Suen, C. Y., see Rokita, J.

Sugimoto, A., see Kitani, K. M.

Sugimoto, A., see Kitani, K. M.

Sun, Y.-N., see Kuo, W.-F.

Talamo, O., see Calabrese, A.

Tang, C.-Y., Chou, H.-L., Wu, Y.-L. \& Ding, Y.-H., Robust fundamental matrix estimation using coplanar constraints

Tang, S.-H., see Ma, W.-M.

22 (2008) 639-665

22 (2008) 1045-1057

22 (2008) 1045-1057

22 (2008) 601-616

22 (2008) 153-168

22 (2008) 1141-1169

22 (2008) 411-429

22 (2008) 1343-1362

22 (2008) 1621-1646

22 (2008) 1403-1425

22 (2008) 1059-1072

22 (2008) 783-805

22 (2008) 195-206

22 (2008) 667-690

22 (2008) 77-93

Tian, X.-D., see Ha, M.-H.

Traoré, I., see Nazar, A.

22 (2008) 461-495

Tsai, T., Huang, Y.-P. \& Chiang, T.-W., A fast two-stage content-based image retrieval approach in the DCT domain

22 (2008) 765-781

Tsang, E. C. C., see Chan, A. P. F.

22 (2008) 137-151

Tsang, E. C. C., see Ng, W. W. Y.

22 (2008) 121-135 
Tsotsos, J. K., see Rothenstein, A. L.

22 (2008) 861-881

Tziritas, G., see Panagiotakis, C.

22 (2008) 1187-1213

Valitutti, A., see Stock, O.

22 (2008) 1045-1057

Vento, M., see Foggia, P.

22 (2008) 843-860

Vitrià, J., see Raducanu, B.

22 (2008) 899-913

Wang, C.-J., Yen, S.-H. \& Wang, P. S., A multimedia

watermarking technique based on SVMs

22 (2008) 1487-1511

Wang, D., see Jiang, L.

22 (2008) 1121-1140

Wang, H.-B., see Xiao, J.-Z.

22 (2008) 183-194

Wang, H.-R., see Xiao, J.-Z.

22 (2008) 183-194

Wang, J.-F., Lin, C.-L., Yen, C.-W., Chang, Y.-H., Chen, T.-Y., Su, K.-P. \& Nagurka, M. L., Determining the association between dermatoglyphics and schizophrenia by using fingerprint asymmetry measures

Wang, J.-H., see Liu, J.-M.

22 (2008) 601-616

22 (2008) 169-181

Wang, K., see Ma, W.-M.

22 (2008) 195-206

Wang, L., Yao, H. \& Cheng, H. D., Effective and automatic calibration using concentric circles

22 (2008) 1379-1401

Wang, P. S., see Wang, C.-J.

22 (2008) 1487-1511

Wang, P. S. P., see Feng, X.

22 (2008) 617-638

Wang, P. S. P., see Lee, S.-W.

22 (2008) 389-410

Wang, P. S. P., see Luo, Y.

22 (2008) 555-584

22 (2008) 445-459

22 (2008) 515-534

Wang, P. S. P., see Shih, F. Y.

22 (2008) 1215-1240

22 (2008) 153-168

Wang, X., see Chen, Q.

Wang, X.-Z., Lu, S.-X. \& Zhai, J.-H., Fast fuzzy multicategory SVM based on support vector domain description

22 (2008) 109-120

Wendling, L., see Rendek, J.

Woo, W., see Yun, J.

22 (2008) 207-232

22 (2008) 497-514

Wu, C., see Jin, F.

22 (2008) 65-75

Wu, J., see Liu, W.

22 (2008) 1587-1598

Wu, J., see Zhou, H.

22 (2008) 279-294

Wu, Y., see Feng, X.

22 (2008) 617-638

$\mathrm{Wu}, \mathrm{Y}$., see You, Q.

22 (2008) 1283-1299

Wu, Y.-L., see Tang, C.-Y.

22 (2008) 783-805

Xiao, J.-Z., Wang, H.-R. \& Wang, H.-B., Knowledge based robotic control against saturation of actuators owing to fuzzy rules

22 (2008) 183-194

Yang, G. \& Zhang, H., Optimal image multiscale mosaic method based on fuzzy integral

22 (2008) 3-15

Yang, J., see Zhang, T.

22 (2008) 667-690

Yang, J. Y., see Chen, Y.

22 (2008) 747-763 
Yang, M. Q., see Chen, Y.

22 (2008) 747-763

Yang, X., see Bai, X.

Yanushkevich, S. N., see Lee, S.-W.

22 (2008) 733-746

Yao, H., see Wang, L.

22 (2008) 389-410

22 (2008) 1379-1401

Yao, Y., see Chen, Q.

22 (2008) 153-168

Ye, D., see Liu, W.

22 (2008) 1587-1598

Yen, C.-W., see Wang, J.-F.

22 (2008) 601-616

Yen, S.-H., see Wang, C.-J.

22 (2008) 1487-1511

Yeung, D. S., see Chan, A. P. F.

22 (2008) 137-151

Yeung, D. S., see Ng, W. W. Y.

22 (2008) 121-135

You, Q., Zheng, N., Gao, L., Du, S. \& Wu, Y., Analysis of solution for supervised graph embedding

Yu, D., see Bai, X.

22 (2008) 1283-1299

22 (2008) 733-746

Yu, M., see Lu, Y.

22 (2008) 55-64

Yuan, K., see Liu, W.

22 (2008) 1587-1598

Yue, H., see Gao, X.

22 (2008) 1447-1460

Yue, P.-X., see Li, F.-C.

22 (2008) 17-32

Yun, J., Abowd, G. D., Ryu, J. \& Woo, W., User identification with user's stepping pattern over the UbiFloorII

Zhai, J.-H., see Wang, X.-Z.

Zhang, C., Ruan, Q. \& Jin, Y., Fusing global and local complete linear discriminant features by fuzzy integral for face recognition

22 (2008) 1427-1445

Zhang, H., see Jiang, L.

22 (2008) 1121-1140

22 (2008) 3-15

Zhang, T., Li, X., Tao, D. \& Yang, J., Local coordinates alignment (LCA): a novel manifold learning approach Zhao, X.-R., see He, Q.

22 (2008) 667-690

22 (2008) 95-108

Zhao, Y., see Ni, F.

22 (2008) 1647-1668

Zheng, J.-W., see Liu, M.-S.

22 (2008) 33-54

Zheng, N., see You, Q.

22 (2008) 1283-1299

Zhou, H., Li, X., Liu, T., Lin, F., Pang, Y., Wu, J., Dong, J. \& $\mathrm{Wu}, \mathrm{J} .$, Recovery of nonrigid structures from 2D observations

22 (2008) 279-294

Zitar, R. A., see Al-Tahrawi, M. M.

22 (2008) 295-322 